\title{
Access tetracoordinate boron-doped polycyclic aromatic hydrocarbons with aggregation-induced emission under mild conditions
}

\author{
Long Jiang, ${ }^{a}$ Dehui Tan, ${ }^{a}$ Xiaobin Chen, ${ }^{a}$ Tinghao Ma, ${ }^{a}$ Baoliang Zhang *a and Deng-Tao Yang *a \\ School of Chemistry and Chemical Engineering, Northwestern Polytechnical University, Xi'an, Shaanxi 710072, \\ China.E-mail: blzhang@nwpu.edu.cn, dtyang@nwpu.edu.cn.
}

\begin{abstract}
The boron-doped polycyclic aromatic hydrocarbons (PAHs) have attracted ongoing attention in the field of optoelectronic materials due to their unique optical and redox properties. To investigate the effect of tetracoordinate boron in PAHs bearing $\mathrm{N}$-heterocycles (indole and carbazole), a facile approach to fourcoordinate boron-doped PAHs was developed, which does not require elevated temperature and presynthesized functionalized boron reactants. Five tetracoordinate boron-doped PAHs (NBNN-1 - NBNN-5) were synthesized with different functional groups. Two of them (NBNN-1 and NBNN-2) could further undergo oxidative coupling reactions to form fused off-plane tetracoordinate boron-doped PAHs NBNN-1f and NBNN2f. Compared to the three-coordinate boron-doped counterparts, the UV/Vis absorption and fluorescent emission are significantly red-shift. Unlike the distinct impact of coordination number of boron on optoelectronic properties, the difference of functional groups on the boron atom has negligible impact on their optical and electrochemical properties. The compounds NBNN-1f and NBNN-2f show aggregation-induced emission.
\end{abstract}

\section{Introduction}

Chemically doping heteroatoms into aromatic compounds has proven to be one of the most effective methods to tune their optoelectronic properties and intermolecular interactions. ${ }^{1}$ Due to the isoelectronic relationship between boron and carbon cation as well as between boron-nitrogen (BN) unit and two carbon atoms (CC) motif, boron becomes an important element that can induce novel and complementary properties. ${ }^{2} \mathrm{BN}$ doped aromatic compounds have a long history of evolution since the first example of $\mathrm{BN}$-arene was reported by Alfred Stock in $1926 .{ }^{3}$ Much the same to many other topics of chemistry, not much attention was paid until the new millennium. Nowadays, the synthesis of BN-doped arenes has become one of the most popular topics in organic chemistry and material chemistry, and numerous new BN-doped aromatic compounds have been synthesized and demonstrated to have very distinct properties in optoelectronic materials. ${ }^{4}$ Representative applications of B-doped polycyclic aromatic hydrocarbons (PAHs) have been demonstrated in organic fieldeffect transistor (OFETs) ${ }^{5}$ and organic light emitting diodes (OLEDs). ${ }^{6}$ Particularly, since PAHs decorated by para-positioned boron and nitrogen atoms has been reported to show thermally activated delayed fluorescence (TADF) with narrow full-width at half-maximum (FWHM) of emission based on efficient HOMOLUMO separation by the multiple resonance effect in 2016, , $^{\text {a }}$ BN-doped PAHs have been extensively studied for nextgeneration OLEDs.

With the further development of BN-PAHs chemistry by advances in synthetic methodology and by the application potential of $\mathrm{BN}$-doped $\pi$-conjugated systems, attention to the structure-property relationship has arisen. The location of $\mathrm{BN}$ unit was found to be important to the optoelectronic properties because its location can change whether BN unit could fully conjugate with PAHs. ${ }^{7}$ Although both the number of $\mathrm{BN}$ unit ${ }^{8}$ and different orientations of $\mathrm{BN}$ units ${ }^{9}$ are believed to exhibit non-negligible impact on the optoelectronic properties and intermolecular interactions, only a few of examples have been documented due to the limited synthetic methodology.

In addition, the majority of previous attention has been paid to the tricoordinate boron units in $\mathrm{PAHs},{ }^{10}$ tetracoordinate boron has been viewed more as a functional group instead of a dopant. ${ }^{11}$ In fact, the coordination number of boron atom shows noteworthy impact on the photophysical properties. Until recently, effort to replace the three-coordinate boron of $\mathrm{BN}$ unit with four-coordinate boron has been made (Scheme 1a). The formation/dissociation of four-coordinate boron unit through a dynamic B-N coordination bond in B-PAHs $(\mathbf{A})^{12}$ has been exploited for the solution-processed fabrication of semiconducting films of poorly soluble B-PAHs. Hatakeyama et al reported that tetracoordinate boron-fused helicenes $(\mathbf{B})^{13}$ as

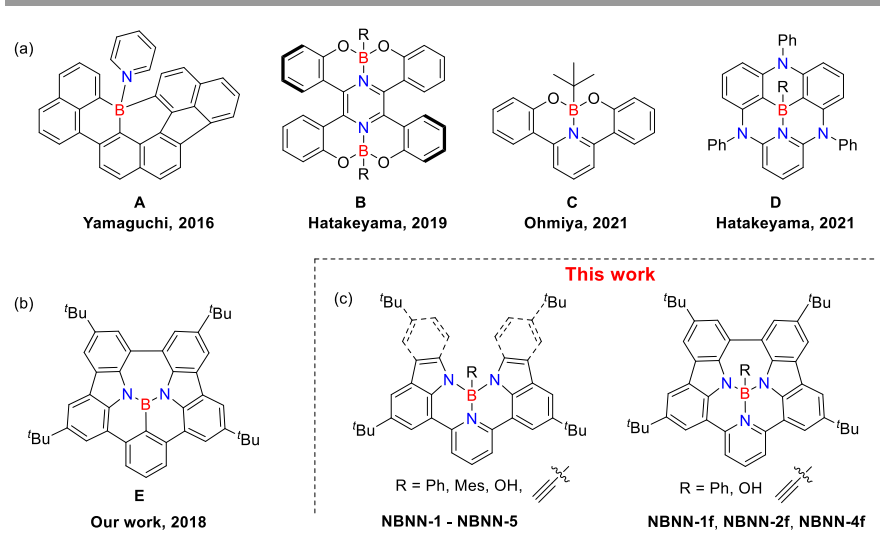

Scheme 1 Representative examples of tetracoordinate B-doped PAHs (a), our previous work (b) and chemical structures of this work (c). 
recently, Hatakeyama et al replaced the tricoordinate boron by tetracoordinate boron to form compound D. ${ }^{15}$

With respect to the synthetic methodology of introducing boron atoms, electrophilic borylation as the symbolic reaction to synthesize borylated compounds has shown advantages in efficiency, scalability and avoidance of previous reagents. However, this type of electrophilic borylation requires high temperature with high boiling point solvents, which needs long reaction time and makes subsequent purification difficult. ${ }^{16}$

We have longstanding interest both in developing synthetic methodology and applications of BN-doped PAHs. ${ }^{17}$ We have developed photo-driven elimination reactions to generate $\mathrm{BN}$ $\mathrm{PAHs}^{18}$ and further expand this elimination using heat. ${ }^{19} \mathrm{We}$ also proposed a new strategy named exciton-driven elimination (EDE) to enable the in situ solid-state conversion of nonemissive tetracoordinate boron-substituted heterocycles to highly emissive BN-PAHs in OLEDs. ${ }^{20}$ In addition, we recently demonstrated combination of electrophilic borylation and oxidative coupling to be a facile and viable approach to new NBN-doped molecular systems with tri-coordinate boron atoms (Scheme 1b). ${ }^{21}$ In this work, we further disclose the strategy of combining electrophilic borylation and oxidative coupling for producing tetra-coordinate boron-doped PAHs. Unlike the typical electrophilic borylation driven by high temperature, our system can quickly undergo borylation at room temperature followed by addition of different nucleophilic reagents to build tetra-coordinate BN-PAHs bearing different functional groups on boron atoms (Scheme 1c).

\section{Results and discussion}

For the synthesis of tetracoordinate boron-doped PAHS NBNN1 - NBNN-5, we started from the commercially available 2,6dibromopyridine, which could undergo Suzuki coupling reaction with readily available 1-Bpin-carbazole and 7-Bpin-indole to produce the precursor compounds $\mathbf{1 a}$ and $\mathbf{1 b}$ in $90 \%$ and $46 \%$ yields, respectively. (Scheme 2 ).

Our first attempt to introduce boron atom using $\mathrm{BBr}_{3}$ as boron source with ${ }^{~} \mathrm{Pr}_{2} \mathrm{NEt}$ in $\mathrm{o}$-dichlorobenzene (o-DCB) at 180 ${ }^{\circ} \mathrm{C}$ was successful to produce phenyl group functionalized $\mathrm{BN}$ PAHs NBNN-1 when phenylmagnesium bromide was used as

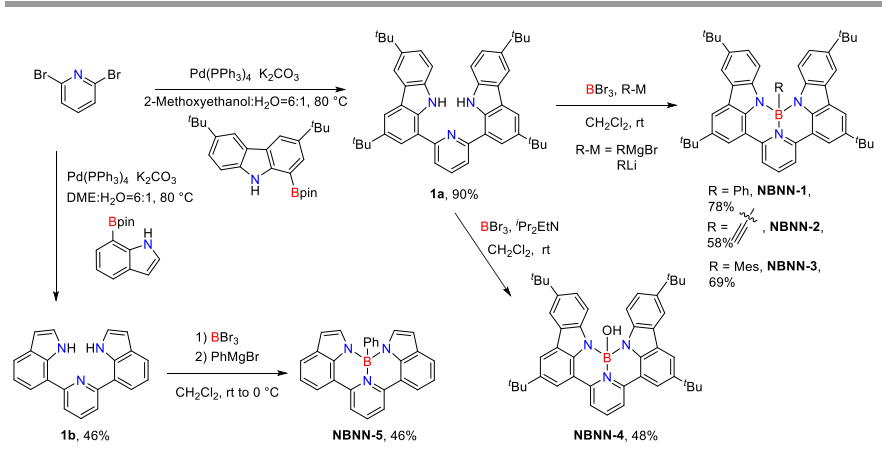

Scheme 2 Synthetic routes for NBNN-1 to NBNN-5
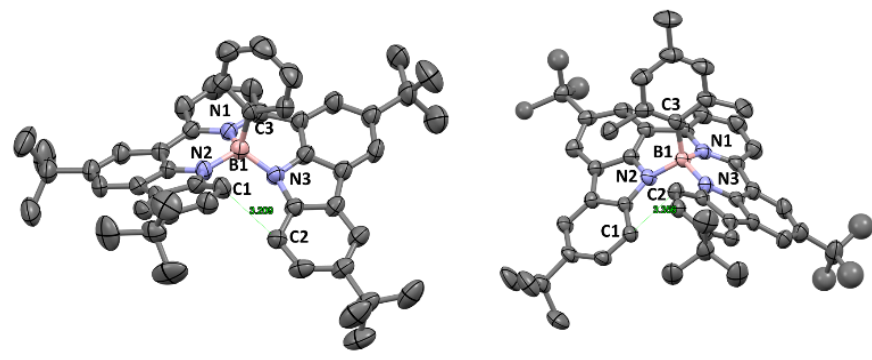

Fig. 1 Left: the crystal structure of NBNN-1. Important bond lengths ( $(\AA)$ : C1-C2 3.209(5), B1-N1 1.653(4), B1-N2 1.508(5), B1-N3 1.541(5), B1-C3 1.614(5). Right the crystal structure of NBNN-3. Important bond lengths (A): C1-C2 3.356(5), B1are set at $50 \%$ probability. Hydrogen atoms omitted for clarity.

the nucleophilic reagent. The existence of $o-D C B$ makes the purification difficult. We tried to use several other solvents with lower boiling points at lower reaction temperatures, all of which generated the target compound NBNN-1 with similar yields around $70 \%$. Usually, the presence of the additive ${ }^{i} \mathrm{Pr}_{2} \mathrm{NEt}$ was proven to be essential to the electrophilic borylation, but absence of ${ }^{i} \mathrm{Pr}_{2} \mathrm{NEt}$ did not show any influence on the borylation yield. To be our delight, the reaction temperature was finally lowered down to room temperature without ${ }^{i} \mathrm{Pr}_{2} \mathrm{NEt}$ in $78 \%$ yield (Scheme 2). In addition, the kind of nucleophiles was also probed, lithium reagent like phenyl lithium could produce compound NBNN-1 in slightly lower yield. While we are preparing the manuscript, the synthesis of NBNN-1 was reported under high temperature. ${ }^{22}$ With the optimal conditions in hands, ethynylmagnesium bromide and mesitylmagnesium bromide were used as nucleophiles to generate compounds NBNN-2 and NBNN-3 in 58\% and 69\% yields, respectively. Compound NBNN-4 was obtained when the reaction was quenched by water without adding any nucleophiles in one digit yield, however, the yield of NBNN-4 was significantly improved to $46 \%$ when ${ }^{i} \mathrm{Pr}_{2} \mathrm{NEt}$ was used to quench the reaction. We also tried other hydroxy group containing reagent like phenol, which did not give the corresponding product at all. Compound NBNN-5 was also synthesized under the optimal conditions. Other nucleophiles like isopropylmagenisium bromide, vinylmagenisium bromide, methyl lithium could also yield the corresponding tetracoordinate boron-doped PAHs, however, those compounds were slowly decomposing on silica gel or alumina. Bulky nucleophile like tbutylmagenisium bromide did not react probably due to the steric hindrance. Compounds NBNN-1 to NBNN-5 were fully characterized by NMR and HRMS spectroscopies. The molecular structures of NBNN-1 and NBNN-3 were further determined by single-crystal X-ray diffraction analysis and shown in Figure 1.

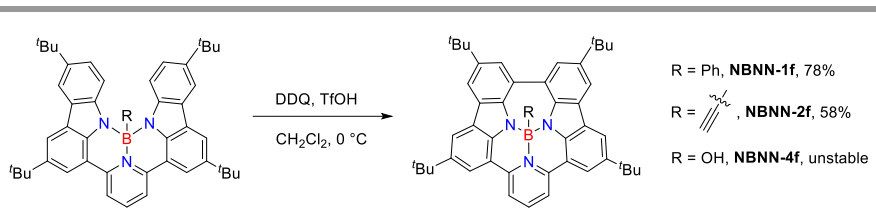

Scheme 3 Formation of fused tetracoordinate boron-doped PAHs via the oxidative coupling 

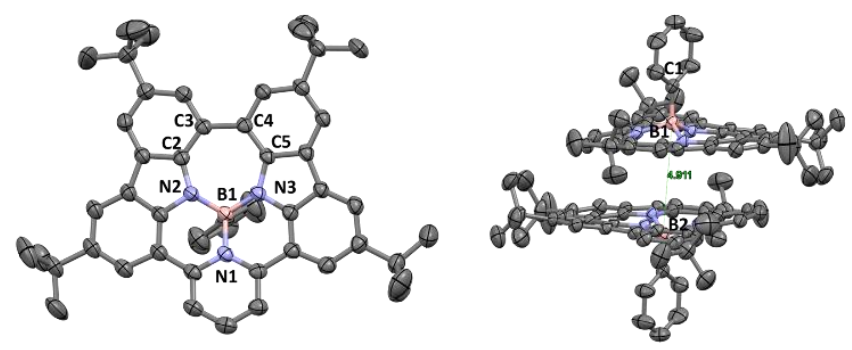

Fig. 2 Left: the crystal structure of NBN-1f (50\% thermal ellipsoids). Hydrogen atoms omitted for clarity. Important bond lengths (A ): B1-B2: 4.911(4), B1-C1 $1.613(4), \mathrm{B} 1-\mathrm{N} 11.640(4), \mathrm{B} 1-\mathrm{N} 21.523(4), \mathrm{B} 1-\mathrm{N} 31.502(3)$
$1.404(4), \mathrm{C} 3-\mathrm{C} 41.510(4), \mathrm{C} 4-\mathrm{C} 51.409(5), \mathrm{C} 5-\mathrm{N} 31.402(3)$.

Similar to our previous work of synthesized tricoordinate borondoped $\mathrm{PAHs},{ }^{21}$ the proximity and the steric repulsion between the hydrogen atoms of flanking units in NBNN-1 to NBNN-4 render them undergo oxidative coupling reaction to form the fused forms. The combination of 2,3-dichloro-5,6-dicyano-1,4benzoquinone (DDQ) as the oxidant and $\mathrm{TfOH}$ as the acid, the fully fused compounds NBNN-1f, NBNN-2f and NBNN-4f were successfully observed. Compound NBNN-1f and NBNN-2f were obtained in isolate yields of $78 \%$ and $58 \%$, respectively (Scheme 3). Silica gel or alumina column chromatography was not compatible to NBNN-4f, which decomposed on silica gel or alumina and was confirmed by HRMS. NBNN-3 did not undergo oxidative coupling reaction probably due to the longer distance (3.356 ̊̊, Figure 1) between two carbon atoms. Unlike the tricoordinate boron-doped PAH analogue ( $E$ in Scheme 1$),{ }^{21}$ the crystal structure of NBNN-1f shows that the four coordinated boron atom adopts a tetrahedral geometry, which makes the molecular structure off-plane (Figure 2). The B1-N1, B1-N2, and B1-N3 bond lengths are $1.640 \AA$, $1.523 \AA$, and $1.502 \AA$, respectively. Similar to tricoordinate boron-doped PAH E, the $\pi$ stacking interactions of NBNN-1f are limited to two neighbouring molecules with longer separation distance than $\mathbf{E}$. The absorption, emission and electrochemical data of these seven tetracoordinate boron-doped PAHs are shown in Figure 3 and Table 1. The maximums of absorption of NBNN-1 to NBNN5 are around $460 \mathrm{~nm}$ with the exceptions of NBNN-3 and NBNN5 , in which the absorption peaks are red-shit to $481 \mathrm{~nm}$ and blue-shift to $446 \mathrm{~nm}$, respectively. The absorption peaks are in agreement with the colors of their THF solutions (Figure $3 c$ ).
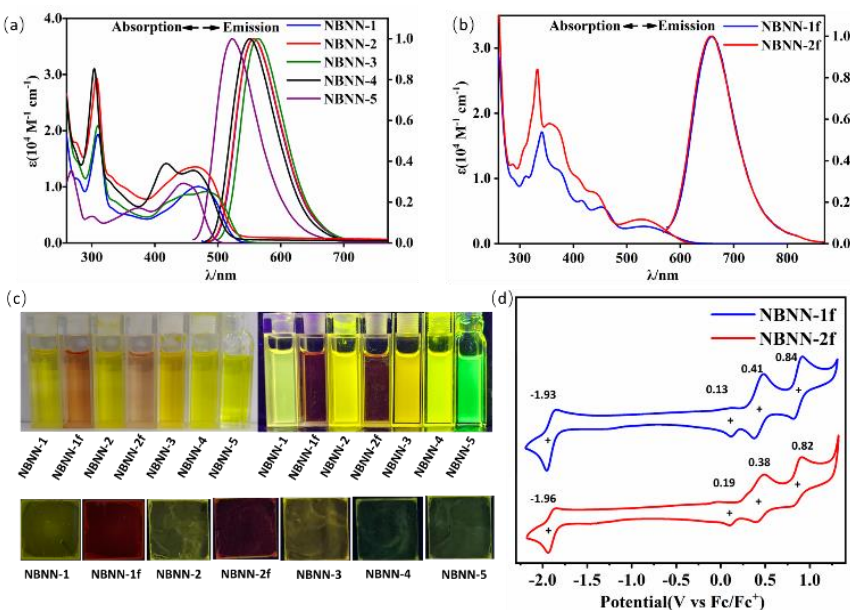

Fig. 3 (a) UV/vis and emission spectra of NBNN-1 to NBNN-5 in THF $\left(1.0 \times 10^{-5} \mathrm{M}\right)$ (b) UV/vis and emission spectra of NBNN-1f to NBNN-2f in THF $(1.0 \times 10-5 \mathrm{M})$ ). (c Photographs showing the colors (top left), fluorescent colors (top right) and PMMA films (bottom) of NBNN-1 to NBNN-5, NBNN-1f and NBNN-2f. (d) Cyclic voltammograms of NBNN-1f and NBNN-2f: glassy carbon as working electrode, $\mathrm{P}$ wire as reference and counter electrodes. $\mathrm{Fc}^{-\mathrm{Fc}^{+}}=$ferrocene/ferrocenium $\mathrm{mV} / \mathrm{s}$.

According to the time-dependent density functional theory (TD-DFT) calculations (Figure 4, and see Supporting Information (SI) for details), this group of absorption peaks are ascribable to the $\mathrm{S}_{0} \rightarrow \mathrm{S}_{1}$ with transition configuration of HOMO $\rightarrow$ LUMO, while smaller HOMO-LUMO gap results in red-shifted absorption peaks of NBNN-3 and larger HOMO-LUMO gap brings about the blue-shifted absorption peaks of NBNN-5. The strong absorption peaks at $310 \mathrm{~nm}$ of NBNN-1 - NBNN-4 are likely attributed to the larger conjugation of carbazole unit than indole unit in NBNN-5.

Not surprisingly, except NBNN-5, molecules NBNN-1 NBNN-4 show near-identical fluorescence spectra with emissive peaks between $551 \mathrm{~nm}$ and $564 \mathrm{~nm}$, which suggests that the substituents on the boron atom of tetracoordinate borondoped PAHs have negligible contribution on photophysical properties. The fluorescent peaks in solid of NBNN-1 to NBNN4 are very similar to those in THF solution, while the $\lambda_{\mathrm{em}}$ of NBNN-5 in solid was red-shit to $540 \mathrm{~nm}$ from bright green color to greenish yellow color as shown in Figure 3c. The quantum yields both in solution and in poly (methyl methacrylate) (PMMA) film of the open form of compounds bearing carbazole units are $25 \%$, except NBNN-1 which have much lower quantum yields.

Table 1 Photophysical and electrochemical data of NBNN-1 to NBNN-5, NBNN-1f and NBNN-2f

\begin{tabular}{|c|c|c|c|c|c|c|c|c|}
\hline & \multicolumn{2}{|c|}{ UV/vis ${ }^{a}$} & \multicolumn{2}{|c|}{ Fluorescence in THF solution ${ }^{a}$} & \multicolumn{2}{|c|}{ Fluorescence in PMMA film ${ }^{b}$} & \multicolumn{2}{|c|}{ Electrochemical data $^{c}$} \\
\hline & $\lambda_{a b s}(\mathrm{~nm})$ & $\varepsilon\left(10^{-4} \mathrm{M}^{-1} \mathrm{~cm}^{-1}\right)$ & $\lambda_{e m}(\mathrm{~nm})$ & $\Phi^{d}(\%)$ & $\lambda_{e m}(\mathrm{~nm})$ & $\Phi^{d}(\%)$ & $E_{\text {red }}(V)$ & $\mathrm{E}_{o x}(\mathrm{~V})$ \\
\hline NBNN-1 & 469 & 1.00 & 557 & 8.8 & 556 & 15.7 & -2.02 & 0.30 \\
\hline NBNN-1f & 531 & 0.27 & 660 & 0.8 & 621 & 4.0 & -1.93 & 0.13 \\
\hline NBNN-2 & 465 & 1.36 & 558 & 23.4 & 562 & 22.6 & -2.16 & 0.26 \\
\hline NBNN-2f & 528 & 0.37 & 659 & 0.8 & 624,659 & 4.0 & -1.96 & 0.19 \\
\hline NBNN-3 & 481 & 0.91 & 564 & 24.5 & 561 & 26.6 & -2.09 & 0.27 \\
\hline NBNN-5 & 446 & 1.06 & 523 & 8.3 & 540 & 9.5 & $-^{e}$ & $-e$ \\
\hline
\end{tabular}

${ }^{a} 10^{-5} \mathrm{M}$ in THF at $298 \mathrm{~K} .{ }^{b}$ PMMA film (10 wt\%). ${ }^{c}$ vs. Fc/Fc ${ }^{+}$(ferrocene/ferrocenium) glassy carbon as working electrode, $\mathrm{Pt}$ wire as reference and counter electrodes, ferrocene $\left(\mathrm{Fc} / \mathrm{Fc}^{+}=\right.$ferrocene/ferrocenium) was added to the solution as the internal reference, scan rate $=100 \mathrm{mV} / \mathrm{s}$. ${ }^{d}$ Quantum yields were determined using a calibrated integrating sphere. ${ }^{e}$ Cyclic voltammogram of NBNN-5 did not show resolved redox peaks (see SI for details) 


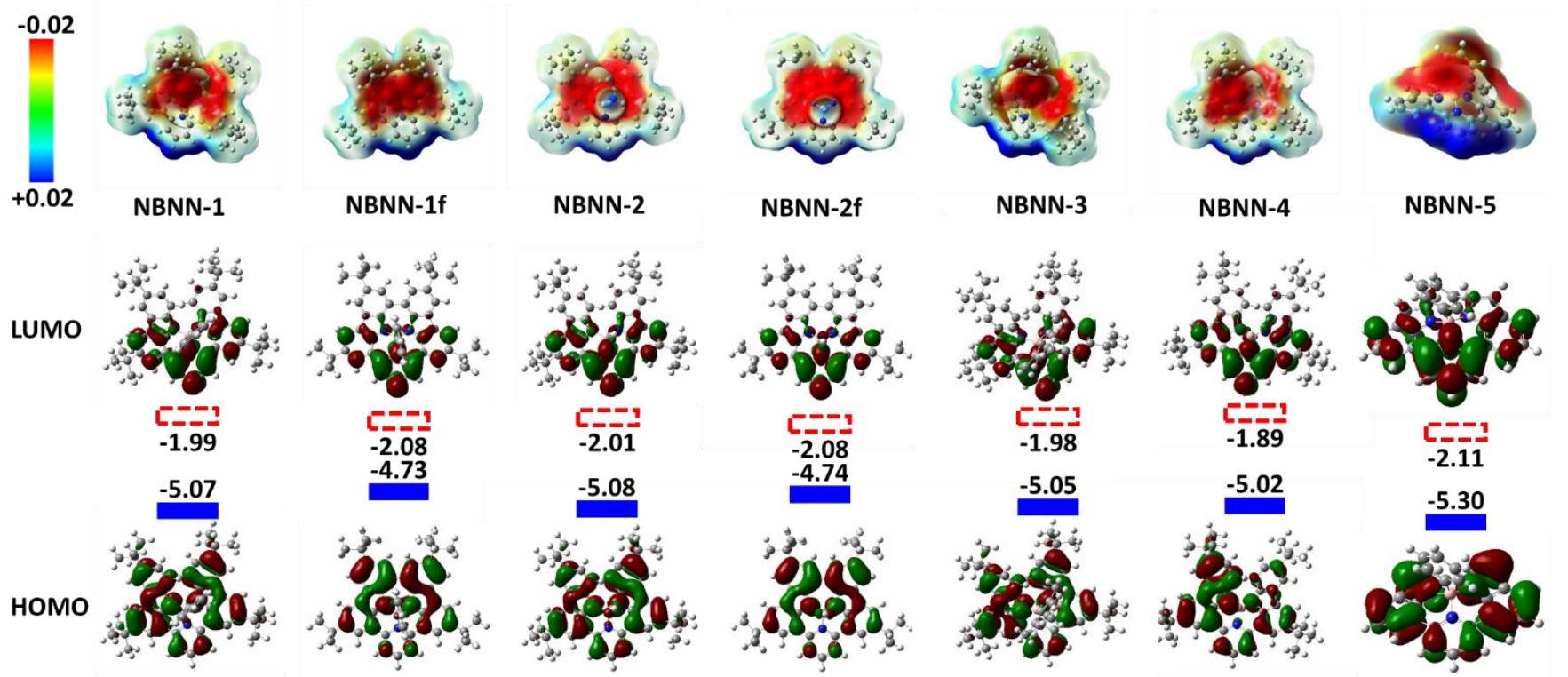

Fig. 4 Top: electrostatic potentials of tetracoordinate boron-doped PAHs NBNN-1 - NBNN-5, NBNN-1f, and NBNN-2f. Bottom: DFT calculated energies and diagrams of HOMO and LUMO for NBNN-1 - NBNN-5, NBNN-1f, and NBNN-2f at the B3LYP/6-31g(d) level.

The compound NBNN-5 bearing indole units only has less than $10 \%$ quantum yield both in solution and in solid. Compared to NBNN-1 and NBNN-2, the emission peaks of the fused molecules NBNN-1f and NBNN-2f show remarkable red-shift up to $100 \mathrm{~nm}$ and appear indistinguishable fluorescent spectra $\left(\lambda_{\text {em }}\right.$ $=660 \mathrm{~nm}, \Phi<1 \%$ ), while their absorption spectra are also similar with different absorption extinction coefficients. The fluorescence peak of NBNN-1f in PMMA film was blue-shifted up to $39 \mathrm{~nm}$ compared to that in solution (see SI for details), while NBNN-2f in PMMA film exhibits two emission peaks at $624 \mathrm{~nm}$ and $659 \mathrm{~nm}$. Both NBNN-1f and NBNN-2f show significant increase on quantum yields in solid up to $4 \%$. The low quantum yields of NBNN-1f and NBNN-2f are likely due to the internal conversion between small $\mathrm{S}_{0}-\mathrm{S}_{1}$ gaps. The fluorescence peaks in solution and solid are consistent with the emission colors of their THF solutions and PMMA films (Figure 3c).

Molecules NBNN-1 to NBNN-5 display pseudo-reversible reduction peaks at $\sim-2.0 \mathrm{~V}\left(\mathrm{vs} . \mathrm{Fc} / \mathrm{Fc}^{+}\right)$and multiple reversible oxidation peaks with the first oxidation peaks at $\sim 0.3 \mathrm{~V}$ (vs.Fc/Fc ${ }^{+}$) (Table 1 and SI). The fused forms of boron-doped PAHs NBNN-1f and NBNN-2f show almost identical cyclic voltammograms as shown in Figure 3d. The first oxidation peaks of NBNN-1f and NBNN-2f are lower than those of NBNN-1 and NBNN-2, which can be attributed to the high-lying HOMO level (Figure 4 bottom). The experimental data are in good agreement with calculated HOMO and LUMO. The fact that the substituents on the boron atom does not affect their electrochemical properties indicates the four-coordinate boron atom is responsible to redox properties. Our previous study on tricoordinate boron-PAHs did not show such rich electrochemical properties. ${ }^{21}$ Therefore, imbedding tetracoordinate boron atom into PAHs is regarded as an effective approach to enrich the redox properties of PAHs.

Unlike the tricoordinate boron-doped PAHs analogues in which the boron atom has a significant contribution to LUMO and the HOMO/LUMO spread over the entire molecules, ${ }^{21}$ the four-coordinate boron atom in NBNN-1 to NBNN-5, NBNN-1f and NBNN-2f has no contribution to LUMO (Figure 4), and their LUMOs mainly locate on the pyridine and phenyl rings of carbazole and indole connected to pyridine. Once again, the calculated HOMO and LUMO prove the substituents on fourcoordinate boron atom have trivial adjustment on the frontier molecular orbitals. Compared to NBNN-1 and NBNN-2, NBNN1f and NBNN-2f has smaller HOMO-LUMO gaps due to the notably elevated HOMO and slightly lowered LUMO, which could indicate the reason why the fused product NBNN-4f of NBNN-4 was less stable and slowing decomposed on silica gel or alumina (Scheme 3). We note that the electrostatic potential maps of NBNN-1 - NBNN-5, NBNN-1f and NBNN-2f display the development of negative characters on the tetracoordinate boron area and positive characters on the pyridine unit (Figure 4 top).

To illustrate the aromaticity of these tetracoordinate boron-doped PAHs, the nucleus-independent chemical shift $(\mathrm{NICS}(1))^{23}$ of two C3NBN rings and 7-memebered C4NBN ring (if exists) around boron atoms were calculated (Figure 5a and $\mathrm{SI})$. The two C3NBN rings in NBNN-1 - NBNN-5 has positive NICS(1) values (see SI), supporting their antiaromaticity. The two C3NBN rings in NBNN-1f and NBNN-2f has much more positive NICS(1) values than those of NBNN-1 - NBNN-5, indicating they are more antiaromatic. Similar to the tricoordinate boron-doped $\mathrm{PAH},{ }^{21}$ the 7-membered $\mathrm{C} 4 \mathrm{NBN}$ has a larger NICS(1) value of $13.8 \mathrm{ppm}$ (Figure 5a). The anisotropy of the induced current density (ACID) calculations ${ }^{24}$ that are based on the optimized structures further support the antiaromaticity of two C3NBN rings and C4NBN ring, showing an anti-clockwise diatropic ring current circuit. In addition, the 13-membered ring with a boron center of NBNN-1f is antiaromatic with an anticlockwise diatropic ring current circuit, as shown in Figure 5a.

The fused molecules NBNN-1f and NBNN-2f exhibit aggregation-induced emission (AIE) behavior, ${ }^{25}$ while compounds NBNN-1 - NBNN-5 only have normal 

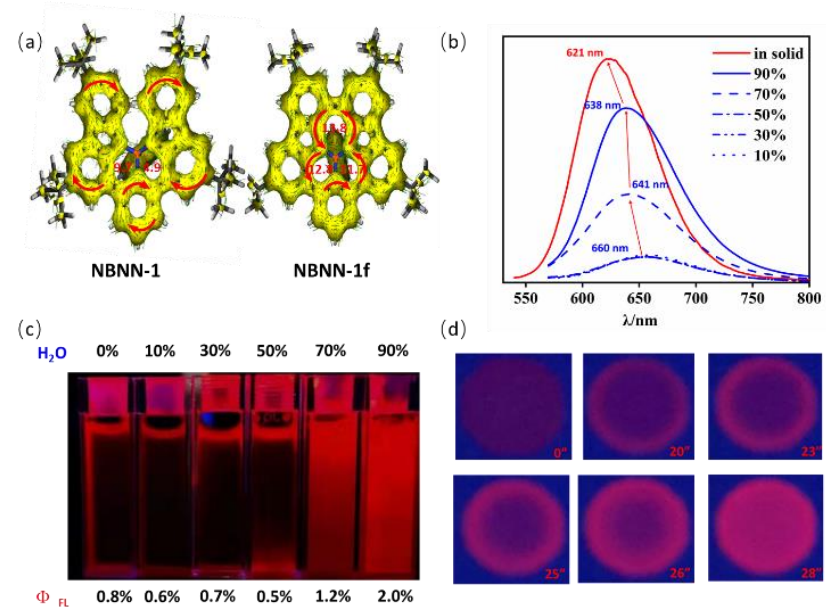

Fig. 5 (a) ACID plots for NBNN-1 and NBNN-1f, numbers in red representing NICS (1) in ppm. (b) Emission spectra change of NBNN-1f in THF with different $\mathrm{H}_{2} \mathrm{O}$ fractions (vol\%). (c) Photographs showing the emission intensity increasing with
$\mathrm{H}_{2} \mathrm{O}$ fraction increasing. (d) Photographs showing one drop of NBNN-1f solution on a thin-layer chromatography plate with different evaporation timescales.

properties. AIE behavior of NBNN-1f and NBNN-2f is probably due to their structure constraint. We used NBNN-1f as an example to investigate the AIE phenomenon in detail (Figure 5b$5 \mathrm{~d})$. The red emission became stronger after the water content in volume reached $50 \%$ in THF, the emission intensity grew faster when water was further added, as shown in Figure $5 \mathrm{~b}$ and $5 \mathrm{c}$. The THF solution with $90 \%$ water displayed bright red emission. The quantum yields gradually increased when water fraction increased. In addition, we found that the AIE behavior of NBNN-1f was accompanied by the blue-shift of emission (Figure 2b). AlE behavior of NBNN-1f was further demonstrated through change of emission intensity after adding one drop of NBNN-1f THF solution on a thin-layer chromatography plate (Figure $5 d$ ). We observed a notable increase of the red emission under 365 nm UV lamp irradiation.

\section{Conclusions}

We developed an efficient strategy to construct a series of tetracoordinate boron-doped PAHs with various substituents on boron atoms at room temperature. Some of them were successfully converted to a fully fused four-coordinate borondoped NBN-PAHs via oxidative coupling. The substituents on boron atoms were found to have negligible impact on photophysical and electrochemical properties. All the rings with tetracoordinate boron atoms are antiaromatic. The fused NBNPAHs display blue-shifted AIE behavior.

\section{Conflicts of interest}

There are no conflicts to declare.

\section{Acknowledgements}

The authors thank the National Natural Science Foundation of China (22001211) and Northwestern Polytechnical University for financial support. This project is also supported by special fund of Shaanxi Key Laboratory of Special Fuel Chemistry and Material (SPCF-SKL-2021-0014).

\section{Notes and references}

\#\#Note: The crystal structures: CCDC 2128933 (NBNN-1), 2128995 (NBNN-1f), 2129008 (NBNN-3)

1 (a) X. Feng, J. Wu, M. Ai, W. Pisula, L. Zhi, J. P. Rabe and K. Müllen, Angew. Chem. Int. Ed., 2007, 46, 3033-3036; (b) M. Takase, V. Enkelmann, D. Sebastiani, M. Baumgarten and K. Müllen, Angew. Chem. Int. Ed., 2007, 46, 5524-5527; (c) J. Wei, B. Han, Q. Guo, X. Shi, W. Wang and N. Wei, Angew. Chem. Int. Ed., 2010, 49, 8209-8213. (d) T. Hatakeyama, S. Hashimoto, S. Seki and M. Nakamura, J. Am. Chem. Soc., 2011, 133, 18614-18617; (e) Z. Zhou, A. Wakamiya, T. Kushida and S. Yamaguchi, J. Am. Chem. Soc., 2012, 134, 4529-4532; (f) X. Wang, F. Zhang, J. Liu, R. Tang, Y. Fu, D. Wu, Q. Xu, X. Zhuang, G. He and X. Feng, Org. Lett., 2013, 15, 5714-5717; (g) L. Zou, X.-Y. Wang, K. Shi, J.-Y. Wang and J. Pei, Org. Lett., 2013, 15, 4378-4381; (h) E. Gońka, P. J. Chmielewski, T. Lis and M. Stępień, J. Am. Chem. Soc., 2014, 136, 16399-16410; ( i) V. M. Hertz, M. Bolte, H.-W. Lerner and M. Wagner, Angew. Chem. Int. Ed., 2015, 54, 8800-8804; (j) H. Oshima, A. Fukazawa, T. Sasamori and S. Yamaguchi, Angew. Chem. Int. Ed., 2015, 54, 7636-7639; (k) M. Hirai, N. Tanaka, M. Sakai and S. Yamaguchi, Chem. Rev., 2019, 119, 8291-8331; (I) N. Ando, T. Yamada, H. Narita, N. N. Oehlmann, M. Wagner and S. Yamaguchi, J. Am. Chem. Soc., 2021, 143, 9944-9951.

2 (a) Z. Liu and T. B. Marder, Angew. Chem. Int. Ed., 2008, 47, 242-244; (b) J. F. Araneda, B. Neue and W. E. Piers, Angew. Chem. Int. Ed., 2012, 51, 9977-9979; (c) J. S. A. Ishibashi, J. L. Marshall, A. Mazière, G. J. Lovinger, B. Li, L. N. Zakharov, A. Dargelos, A. Graciaa, A. Chrostowska and S.-Y. Liu, J. Am. Chem. Soc., 2014, 136, 15414-15421; (d) M. Krieg, F. Reicherter, P. Haiss, M. Ströbele, K. Eichele, M.-J. Treanor, R. Schaub and H. F. Bettinger, Angew. Chem. Int. Ed., 2015, 54, 8284-8286; (e) J. Dosso, J. Tasseroul, F. Fasano, D. Marinelli, N. Biot, A. Fermi and D. Bonifazi, Angew. Chem. Int. Ed., 2017, 56, 4483-4487; (f) S. Limberti, L. Emmett, A. Trandafir, G. Kociok-Köhn and G. D. Pantoş, Chem. Sci., 2019, 10, 95659570; (j) J. Hoffmann, B. Geffroy, E. Jaques, M. Hissler and A. Staubitz, J. Mater. Chem. C., 2021, 9, 14720-14729.

3 A. Stock, E. Pohland, Ber. Dtsch. Chem. Ges., 1926, 59, 2215 $-2223$.

4 (a) M. Fingerle, C. Maichle-Mössmer, S. Schundelmeier, B. Speiser and H. F. Bettinger, Org. Lett., 2017, 19, 4428-4431; (b) D. Shimizu, K. Furukawa and A. Osuka, Angew. Chem. Int. Ed., 2017, 56, 7435-7439; (c) N. Ando, T. Kushida and S. Yamaguchi, Chem. Commun., 2018, 54, 5213-5216; (d) J.-K. Li, X.-Y. Chen, Y.-L. Guo, X.-C. Wang, A. C. H. Sue, X.-Y. Cao and X.-Y. Wang, J. Am. Chem. Soc., 2021, 143, 17958-17963; (e) Wang, X. Fang, X. Guo, Q. Wu, Q. Gong, C. Yu, E. Hao and L. Jiao, Org. Lett., 2021, 23, 4796-4801.

5 (a) X.-Y. Wang, F.-D. Zhuang, X. Zhou, D.-C. Yang, J.-Y. Wang and J. Pei, J. Mater. Chem. C., 2014, 2, 8152-8161; (b) S. K. Mellerup and S. Wang, Trend. Chem., 2019, 1, 77-89; (c) R. Zhao, Y. Min, C. Dou, B. Lin, W. Ma, J. Liu and L. Wang, ACS Appl. Polym. Mater., 2020, 2, 19-25; (d) J. Hoffmann, B. 
Geffroy, E. Jaques, M. Hissler and A. Staubitz, J. Mater. Chem. C., 2021, 9, 14720-14729.

6 (a) T. Hatakeyama, K. Shiren, K. Nakajima, S. Nomura, S. Nakatsuka, K. Kinoshita, J. Ni, Y. Ono and T. Ikuta, Adv. Mater., 2016, 28, 2777-2781; (b) H. Nakanotani, T. Furukawa, T. Hosokai, T. Hatakeyama and C. Adachi, Adv. Opt. Mater., 2017, 5, 1700051; (c) H. Fukagawa, T. Oono, Y. Iwasaki, T. Hatakeyama and T. Shimizu, Mater. Chem. Front., 2018, 2, 704-709; (d) X. Liang, Z.-P. Yan, H.-B. Han, Z.-G. Wu, Y.-X. Zheng, H. Meng, J.-L. Zuo and W. Huang, Angew. Chem. Int. Ed., 2018, 57, 11316-11320; (e) G. Meng, X. Chen, X. Wang, N. Wang, T. Peng and S. Wang, Adv. Opt. Mater., 2019, 7, 1970043; (f) S. K. Mellerup and S. Wang, Trend. Chem., 2019, 1, 77-89. (g) J. U. Kim, I. S. Park, C.-Y. Chan, M. Tanaka, Y. Tsuchiya, H. Nakanotani and C. Adachi, Nat. Commun., 2020, 11, 1765; (h) Y. H. Lee, Y.-S. Shin, T. Lee, J. Jung, J.-H. Lee and M. H. Lee, Chem. Eng. J., 2021, 423, 130224.

7 (a) X.-Y. Wang, F.-D. Zhuang, X.-C. Wang, X.-Y. Cao, J.-Y. Wang and J. Pei, Chem. Commun., 2015, 51, 4368-4371; (b) M. Numano, N. Nagami, S. Nakatsuka, T. Katayama, K. Nakajima, S. Tatsumi, N. Yasuda and T. Hatakeyama, Chem. Eur. J., 2016, 22, 11574-11577; (c) S. K. Mellerup, C. Li, T. Peng and S. Wang, Angew. Chem. Int. Ed., 2017, 56, 60936097; (d) Y. Fu, X. Chang, H. Yang, E. Dmitrieva, Y. Gao, J. Ma, L. Huang, J. Liu, H. Lu, Z. Cheng, S. Du, H.-J. Gao and X. Feng, Angew. Chem. Int. Ed., 2021, 60, 26115-26121; (e) M. Zhao and Q. Miao, Angew. Chem. Int. Ed., 2021, 60, 21289-21294.

8 (a) T. Agou, J. Kobayashi and T. Kawashima, Org. Lett., 2006, 8, 2241-2244; (b) F. Qiu, F. Zhang, R. Tang, Y. Fu, X. Wang, S. Han, X. Zhuang and X. Feng, Org. Lett., 2016, 18, 1398-1401; (c) Z.-C. He, S. K. Mellerup, L. Liu, X. Wang, C. Dao and S. Wang, Angew. Chem. Int. Ed., 2019, 58, 6683-6687; (d) Y. Chen, W. Chen, Y. Qiao, X. Lu and G. Zhou, Angew. Chem. Int. Ed., 2020, 59, 7122-7130; (e) S. M. Suresh, E. Duda, D. Hall, Z. Yao, S. Bagnich, A. M. Z. Slawin, H. Bässler, D. Beljonne, M. Buck, Y. Olivier, A. Köhler and E. Zysman-Colman, J. Am. Chem. Soc., 2020, 142, 6588-6599; (f) D. Shimoyama, N. Baser-Kirazli, R. A. Lalancette and F. Jäkle, Angew. Chem. Int. Ed., 2021, 60, 17942-17946.

9 (a) X. Wang, F. Zhang, J. Liu, R. Tang, Y. Fu, D. Wu, Q. Xu, X. Zhuang, G. He and X. Feng, Org. Lett., 2013, 15, 5714-5717; (b) X. Wang, F. Zhang, J. Gao, Y. Fu, W. Zhao, R. Tang, W. Zhang, X. Zhuang and X. Feng, J. Org. Chem., 2015, 80, 10127-10133; (c)X.-Y. Wang, A. Narita, X. Feng and K. Müllen, J. Am. Chem. Soc., 2015, 137, 7668-7671; (d) Q. Zhang, Z. Sun, L. Zhang, M. Li, L. Zi, Z. Liu, B. Zhen, W. Sun and X. Liu, J. Org. Chem., 2020, 85, 7877-7883; (e) P.-F. Zhang, J.-C. Zeng, F.-D. Zhuang, K.-X. Zhao, Z.-H. Sun, Z.-F. Yao, Y. Lu, X.Y. Wang, J.-Y. Wang and J. Pei, Angew. Chem. Int. Ed., 2021, 60, 23313-23319.I

10 (a) T. Hatakeyama, S. Hashimoto, T. Oba and M. Nakamura, J. Am. Chem. Soc., 2012, 134, 19600-19603; (b) N. Ando, H. Soutome and S. Yamaguchi, Chemical Science, 2019, 10, 7816-7821; (c) J. Wang, N. Wang, G. Wu, S. Wang and X. Li, Angew. Chem. Int. Ed., 2019, 58, 3082-3086; (d) N. BaserKirazli, R. A. Lalancette and F. Jäkle, Angew. Chem. Int. Ed., 2020, 59, 8689-8697; (e) J. Guo, Y. Yang, C. Dou and Y. Wang, J. Am. Chem. Soc., 2021, 143, 18272-18279; (f) F. D. Zhuang,
J. H. Yang, Z. H. Sun, P. F. Zhang, Q. R. Chen, J. Y. Wang and J. Pei, Chin. J. Chem. 2021, 39, 909-912.

11 (a) J. F. Araneda, W. E. Piers, B. Heyne, M. Parvez and R. McDonald, Angew. Chem. Int. Ed., 2011, 50, 12214-12217; (b) J. F. Araneda, W. E. Piers, B. Heyne, M. Parvez and R. McDonald, Angew. Chem. Int. Ed. Engl., 2011, 50, 1221412217; (c) Y. Min, C. Dou, H. Tian, Y. Geng, J. Liu and L. Wang, Angew. Chem. Int. Ed. Engl., 2018, 57, 2000-2004; (d) S. Mula, N. Leclerc, P. Leveque, P. Retailleau and G. Ulrich, J. Org. Chem., 2018, 83, 14406-14418; (e) D. Cappello, R. R. Maar, V. N. Staroverov and J. B. Gilroy, Chem. Eur. J., 2020, 26, 5522-5529.

12 K. Matsuo, S. Saito and S. Yamaguchi, Angew. Chem. Int. Ed. Engl., 2016, 55, 11984-11988.

13 S. Oda, T. Shimizu, T. Katayama, H. Yoshikawa and T. Hatakeyama, Org. Lett., 2019, 21, 1770-1773.

14 Y. Sato, Y. Goto, K. Nakamura, Y. Miyamoto, Y. Sumida and H. Ohmiya, ACS Catal., 2021, 11, 12886-12892.

15 H. Gotoh, S. Nakatsuka, H. Tanaka, N. Yasuda, Y. Haketa, H. Maeda and T. Hatakeyama, Angew. Chem. Int. Ed. Engl., 2021, 60, 12835-12840.

16 (a) S. Nakatsuka, H. Gotoh, K. Kinoshita, N. Yasuda and T. Hatakeyama, Angew. Chem. Int. Ed., 2017, 56, 5087-5090; (b) K. Matsui, S. Oda, K. Yoshiura, K. Nakajima, N. Yasuda and T. Hatakeyama, J. Am. Chem. Soc., 2018, 140, 1195-1198; (c) P. B. Pati, E. Jin, Y. Kim, Y. Kim, J. Mun, S. J. Kim, S. J. Kang, W. Choe, G. Lee, H.-J. Shin and Y. S. Park, Angew. Chem. Int. Ed., 2020, 59, 14891-14895. (d) S. A. Iqbal, J. Pahl, K. Yuan and M. J. Ingleson, Chem. Soc. Rev., 2020, 49, 4564-4591.

17 S. K. Mellerup and S. Wang, Chem. Soc. Rev., 2019, 48, 36743674.

18 J.-S. Lu, S.-B. Ko, N. R. Walters, Y. Kang, F. Sauriol and S. Wang, Angew. Chem. Int. Ed., 2013, 52, 4544-4548.

19 D.-T. Yang, S. K. Mellerup, X. Wang, J.-S. Lu and S. Wang, Angew. Chem. Int. Ed., 2015, 54, 5498-5501.

20 S. Wang, D.-T. Yang, J. Lu, H. Shimogawa, S. Gong, X. Wang, S. K. Mellerup, A. Wakamiya, Y.-L. Chang, C. Yang and Z.-H. Lu, Angew. Chem. Int. Ed., 2015, 54, 15074-15078.

21 D. T. Yang, T. Nakamura, Z. He, X. Wang, A. Wakamiya, T. Peng and S. Wang, Org. Lett., 2018, 20, 6741-6745.

22 G. Meng, L. Liu, Z. He, D. Hall, X. Wang, T. Peng, X. Yin, P. Chen, D. Beljonne, Y. olivier, E. Zysman-Colman, N. Wang and S. Wang, ChemRxiv, 2021. This content is a preprint and has not been peer-reviewed.

23 (a) P. v. R. Schleyer, C. Maerker, A. Dransfeld, H. Jiao and N. J. R. van Eikema Hommes, J. Am. Chem. Soc., 1996, 118, 6317-6318; (b) P. v. R. Schleyer, M. Manoharan, Z.-X. Wang, B. Kiran, H. Jiao, R. Puchta and N. J. R. van Eikema Hommes, Org. Lett., 2001, 3, 2465-2468.

24 (a) R. Herges, D. Geuenich, J. Phys. Chem. A.; 2001, 105, 3214-3220; (b) D. Geuenich, K. Hess, F. Kohler, R. Herges, Chem. Rev.,2005, 105, 3758-3772.

25 (a) J. Luo, Z. Xie, J. W. Y. Lam, L. Cheng, H. Chen, C. Qiu, H. S. Kwok, X. Zhan, Y. Liu, D. Zhu and B. Z. Tang, Chem. Commun., 2001, 1740-1741; (b) J. Mei, N. L. C. Leung, R. T. K. Kwok, J. W. Y. Lam and B. Z. Tang, Chem. Rev., 2015, 115, 1171811940. 